\title{
Bound and ground states for a class of Schrödinger-Poisson systems
}

Fubao Zhang ${ }^{1 *}$ (D) and Li Cai ${ }^{1}$

\author{
"Correspondence: \\ zhangfubao@seu.edu.cn \\ 'school of Mathematics, Southeast \\ University, Nanjing, China
}

\section{Abstract}

We are concerned with the following Schrödinger-Poisson system:

$$
\begin{cases}-\Delta u+u+K(x) \phi u=a(x) u^{3}, & x \in \mathbb{R}^{3}, \\ -\Delta \phi=K(x) u^{2}, & x \in \mathbb{R}^{3} .\end{cases}
$$

Assuming that $K(x)$ and $a(x)$ are nonnegative functions satisfying

$$
\lim _{|x| \rightarrow \infty} a(x)=a_{\infty}>0, \quad \lim _{|x| \rightarrow \infty} K(x)=0,
$$

and other suitable conditions, we show the existence of bound and ground states via a global compactness lemma and the Nehari manifold. Our result extends the existence result of positive solutions for Schrödinger-Poisson system with more than three times growth by Cerami and Vaira (J. Differ. Equ. 248:521-543, 2010) to the system with three times growth.

MSC: $35 \mathrm{~J} 05 ; 35 \mathrm{~J} 50 ; 35 \mathrm{~J} 60$

Keywords: Schrödinger-Poisson system; Variational method; Three times growth; Bound state; Lack of compactness

\section{Introduction and main result}

In this paper we are devoted to the following Schrödinger-Poisson system

$$
\begin{cases}-\Delta u+u+K(x) \phi u=a(x) u^{3}, & x \in \mathbb{R}^{3}, \\ -\Delta \phi=K(x) u^{2}, & x \in \mathbb{R}^{3},\end{cases}
$$

where the potentials $K$ and $a$ satisfy:

$\left(\mathrm{a}_{1}\right) a \in L^{\infty}\left(\mathbb{R}^{3}\right), \lim _{|x| \rightarrow \infty} a(x)=a_{\infty}>0$.

(K) $K \in L^{2}\left(\mathbb{R}^{3}\right), \lim _{|x| \rightarrow \infty} K(x)=0, K(x) \geq 0$, for all $x \in \mathbb{R}^{3}, K \not \equiv 0$.

$\left(\mathrm{a}_{2}\right) a(x) \geq a_{\infty}, \forall x \in \mathbb{R}^{3}, a(x)-a_{\infty}>0$ on a positive measure set.

$\left(\mathrm{a}_{3}\right) a(x) \leq a_{\infty}, \forall x \in \mathbb{R}^{3}, \inf _{x \in \mathbb{R}^{3}} a(x)>0$.

(c) The Author(s) 2019. This article is distributed under the terms of the Creative Commons Attribution 4.0 International License (http://creativecommons.org/licenses/by/4.0/), which permits unrestricted use, distribution, and reproduction in any medium, provided you give appropriate credit to the original author(s) and the source, provide a link to the Creative Commons license, and indicate if changes were made. 
System (SP) is a special form of the Schrödinger-Poisson system

$$
\begin{cases}-\Delta u+V(x) u+K(x) \phi u=f(x, u), & x \in \mathbb{R}^{3}, \\ -\Delta \phi=K(x) u^{2}, & x \in \mathbb{R}^{3},\end{cases}
$$

which has been first introduced in [5] as a physical model describing a charged wave, interacting with its own electrostatic field in quantum mechanics. The unknowns $u$ and $\phi$ represent the wave functions associated to the particle and electric potential, the functions $V$ and $K$ are external potentials, and the nonlinearity $f(x, u)$ simulates the interaction between many particles or external nonlinear perturbations. For more information on the physical aspects, we refer the reader to [5].

There have been many works dealing with (1.1) and the existence of bound and ground states, radial and nonradial solutions, sign-changing solutions, and semiclassical states. Here we mainly are interested in the existence results of bound and ground states. It is well known that the main difficulty of problem (1.1) is the lack of compactness for Sobolev's embedding theorem on the whole space $\mathbb{R}^{3}$. To recover the compactness, many studies were focused on the autonomous case (see [4, 9-11, 14, 17], for example) or on the radially symmetric function space which possesses compact embedding (see [3, 15, 17], for instance). When the potentials are neither constants nor radially symmetric, system (1.1) has also been increasingly receiving interest in recent years, for example, see [1-3,13]. Azzollini and Pomponio [4] considered the existence of ground states of (1.1) when $V$ is nonconstant and possibly unbounded below, $K=1, f(x, u)=|u|^{p-1} u$ and $f(x, u)=u^{5}+|u|^{p-1} u$ with $3<p<5$. In [23], Zhao and Zhao established the existence of ground states of (1.1) with pure power nonlinearity $f(u)$ and $V$ being weakly differentiable, $K=1$. Later, Cerami and Vaira [8] considered the system

$$
\begin{cases}-\Delta u+u+K(x) \phi u=a(x)|u|^{p-1} u, & x \in \mathbb{R}^{3}, \\ -\Delta \phi=K(x) u^{2}, & x \in \mathbb{R}^{3},\end{cases}
$$

where $3<p<5$. Assume that the potential $K(x)$ satisfies condition $(\mathrm{K})$ and $a(x)$ satisfies

$\left(\mathrm{a}_{1}^{\prime}\right) \lim _{|x| \rightarrow \infty} a(x)=a_{\infty}>0, \alpha(x):=a(x)-a_{\infty} \in L^{\frac{6}{5-p}}\left(\mathbb{R}^{3}\right) ;$

they discussed the existence of ground and bound states. More precisely, when $\left(a_{1}^{\prime}\right),\left(a_{2}\right)$, (K) and other suitable restrictions on $K(x)$ and $a(x)$ are satisfied, they showed that problem (1.2) has a ground state by the standard method of Nehari manifold and compactness lemma. Moreover, when $\left(\mathrm{a}_{1}^{\prime}\right),\left(\mathrm{a}_{3}\right),(\mathrm{K})$, and certain restrictions on $K(x)$ and $a(x)$ are satisfied, they proved that system (1.2) possesses a bound state via the linking theorem. After that some researchers focused on problem (1.2). Sun et al. in [19] generalized the existence results of ground states to the asymptotically linear case. In [13], the authors dealt with the equation system

$$
\begin{cases}-\Delta u+V(x) u+K(x) \phi u=a(x)|u|^{p-1} u, & x \in \mathbb{R}^{3}, \\ -\Delta \phi=K(x) u^{2}, & x \in \mathbb{R}^{3},\end{cases}
$$

where $3<p<5$, and they obtained a ground state of (1.3) by imposing some conditions on the decay rate of the potentials. On the other hand, under suitable assumptions on the 
decay rate of the potentials, Cerami and Molle [6] proved the existence of bound states of (1.3). Zhang et al. [22] considered system (1.3) with critical growth, namely

$$
\begin{cases}-\Delta u+V(x) u+K(x) \phi u=u^{5}, & x \in \mathbb{R}^{3}, \\ -\Delta \phi=K(x) u^{2}, & x \in \mathbb{R}^{3} .\end{cases}
$$

When $V \in L^{\frac{3}{2}}\left(\mathbb{R}^{3}\right), K \in L^{2}\left(\mathbb{R}^{3}\right)$ are nonnegative functions, and $|V|_{\frac{3}{2}}+|K|_{2}$ is suitably small, they showed that (1.4) has a bound state via a linking theorem.

It seems that the existence of bound and ground states for problem (1.2) with $p=3$, namely system (SP), has not been studied. So in this paper we will fill this gap. Compared with [8], the main difficulty is the lack of the higher-order term and the competing effect of the nonlocal term with three times growth term. On the one hand, due to the lack of the higher-order term, the standard method of Nehari manifold is invalid. Inspired by [12], by restricting the functional in a set, this functional has a unique maximum point along the nontrivial direction $u$ in $H^{1}\left(\mathbb{R}^{3}\right)$. Then we use the one-to-one correspondence of the functionals on the manifold and an open set of the unit sphere to establish the new method of Nehari manifold. On the other hand, the competing effect of the nonlocal term $K(x) \phi u$ and three times growth term $a(x) u^{3}$ makes some estimations and verifications more complex.

Since we have no symmetry assumptions, similar to [8] we shall recover the compactness of PS sequences by the problem at infinity:

$$
-\Delta u+u=a_{\infty} u^{3} .
$$

$(\mathrm{NSE})_{\infty}$

In order to state the main results, we give some notations as follows. Denote by $w$ the unique radial solution of $(\mathrm{NSE})_{\infty}$, and set $m_{\infty}=\frac{1}{4}\|w\|^{2}$, where $\|\cdot\|$ is the standard norm of $H^{1}\left(\mathbb{R}^{3}\right)$.

In addition, when $\left(\mathrm{a}_{2}\right)$ holds, the problem $-\Delta u+u=a(x) u^{3}$ possesses a ground state $w_{a}$, whose energy is $m_{a}=\frac{1}{4}\left\|w_{a}\right\|^{2}<m_{\infty}$. We denote by $S$ and $\bar{S}$ the best constants for the embeddings of $H^{1}\left(\mathbb{R}^{3}\right)$ and $D^{1,2}\left(\mathbb{R}^{3}\right)$ in $L^{6}\left(\mathbb{R}^{3}\right)$, respectively. Our results are as follows:

Theorem 1.1 Let $\left(\mathrm{a}_{1}\right),\left(\mathrm{a}_{2}\right)$ and $(\mathrm{K})$ hold. Furthermore, assume either

$$
|K|_{2}^{2}<\frac{\left(m_{\infty}-m_{a}\right) \bar{S}^{2} S^{4}}{4 m_{a} m_{\infty}}
$$

or

$$
\int_{\mathbb{R}^{3}} K(x) \phi_{w} w^{2} d x<\int_{\mathbb{R}^{3}}\left(a(x)-a_{\infty}\right) w^{4} d x,
$$

then system (SP) has a ground state.

Theorem 1.2 Let $\left(\mathrm{a}_{1}\right),\left(\mathrm{a}_{3}\right)$ and $(\mathrm{K})$ hold. In addition, assume

$$
|K|_{2}^{2}<\left(\frac{\inf _{x \in \mathbb{R}^{3}} a(x)}{a_{\infty}}-\frac{1}{2}\right) \frac{\bar{S}^{2} S^{4}}{4 m_{\infty}},
$$

then the system (SP) has a bound state. 
The paper is organized as follows. In Sect. 2, we give some preliminaries. In Sect. 3, we introduce the variational setting. In Sects. 4 and 5, we prove Theorems 1.1 and 1.2, respectively.

\section{Notations and preliminaries}

In this paper we use the following notations:

$H^{1}\left(\mathbb{R}^{3}\right)$ is the Sobolev space with standard norm

$$
\|u\|^{2}=\int_{\mathbb{R}^{3}}\left(|\nabla u|^{2}+u^{2}\right) d x
$$

$S_{1}=\left\{u \in H^{1}\left(\mathbb{R}^{3}\right):\|u\|^{2}=1\right\} ; D^{1,2}\left(\mathbb{R}^{3}\right)$ is the Sobolev space endowed with the scalar product and norm

$$
(u, v)_{D^{1,2}}=\int_{\mathbb{R}^{3}} \nabla u \cdot \nabla v d x, \quad\|u\|_{D^{1,2}}^{2}=\int_{\mathbb{R}^{3}}|\nabla u|^{2} d x .
$$

The norm in $L^{r}\left(\mathbb{R}^{3}\right)(1 \leq r \leq \infty)$ is denoted by $|\cdot|_{r} ; S$ and $\bar{S}$ are the best Sobolev constants for the embeddings of $H^{1}\left(\mathbb{R}^{3}\right)$ and $D^{1,2}\left(\mathbb{R}^{3}\right)$ in $L^{6}\left(\mathbb{R}^{3}\right)$, respectively, namely

$$
S=\inf _{u \in H^{1}\left(\mathbb{R}^{3}\right) \backslash\{0\}} \frac{\|u\|}{|u|_{6}}, \quad \bar{S}=\inf _{u \in D^{1,2}\left(\mathbb{R}^{3}\right) \backslash\{0\}} \frac{\|u\|_{D^{1,2}}}{|u|_{6}} .
$$

Without loss of generality, in the sequel, we assume that $a_{\infty}=1$.

System (SP) can be easily transformed into a Schrödinger equation with a nonlocal term. Actually, for all $u \in H^{1}\left(\mathbb{R}^{3}\right)$, consider the linear functional $L_{u}$ defined in $D^{1,2}\left(\mathbb{R}^{3}\right)$ by

$$
L_{u}(v)=\int_{\mathbb{R}^{3}} K(x) u^{2} v d x .
$$

By Hölder and Sobolev inequalities, we have

$$
\left|L_{u}(v)\right| \leq|K|_{2}|u|_{6}^{2}|v|_{6} \leq \bar{S}^{-1}|K|_{2}|u|_{6}^{2}\|v\|_{D^{1,2}}
$$

Hence, the Lax-Milgram theorem implies that there exists a unique $\phi_{u} \in D^{1,2}\left(\mathbb{R}^{3}\right)$ such that

$$
\left(\phi_{u}, v\right)_{D^{1,2}}=L_{u}(v)=\int_{\mathbb{R}^{3}} K(x) u^{2} v d x, \quad \forall v \in D^{1,2}\left(\mathbb{R}^{3}\right) .
$$

Namely, $\phi_{u}$ is the unique solution of $-\Delta \phi=K(x) u^{2}$, and

$$
\phi_{u}(x)=\frac{1}{4 \pi} \int_{\mathbb{R}^{3}} \frac{K(y)}{|x-y|} u^{2}(y) d y .
$$

Substituting $\phi_{u}$ into the first equation of (SP), we get

$$
-\Delta u+u+K(x) \phi_{u} u=a(x) u^{3} .
$$

By (2.1) and (2.2), we obtain

$$
\left\|\phi_{u}\right\|_{D^{1,2}}=\left\|L_{u}\right\|_{\mathcal{L}\left(D^{1,2}\left(\mathbb{R}^{3}\right), \mathbb{R}\right)} \leq \bar{S}^{-1} S^{-2}|K|_{2}\|u\|^{2} .
$$


Then

$$
\left|\int_{\mathbb{R}^{3}} K(x) \phi_{u} u^{2} d x\right| \leq \bar{S}^{-2} S^{-4}|K|_{2}^{2}\|u\|^{4} .
$$

In addition, one easily has that the functional

$$
I(u)=\frac{1}{2}\|u\|^{2}+\frac{1}{4} \int_{\mathbb{R}^{3}} K(x) \phi_{u} u^{2} d x-\frac{1}{4} \int_{\mathbb{R}^{3}} a(x) u^{4} d x
$$

is of class $C^{1}$ and its critical points are solutions of (SP)'.

Define the operator $\Phi: H^{1}\left(\mathbb{R}^{3}\right) \rightarrow D^{1,2}\left(\mathbb{R}^{3}\right)$ as

$$
\Phi(u)=\phi_{u}
$$

Below we summarize some properties of $\Phi$, which were proved in $[6,8]$.

\section{Lemma 2.1}

(1) $\Phi$ is continuous;

(2) $\Phi$ maps bounded sets into bounded sets;

(3) $\Phi(t u)=t^{2} \Phi(u)$ for all $t \in \mathbb{R}$;

(4) If $u_{n} \rightarrow u$ in $H^{1}\left(\mathbb{R}^{3}\right)$, then $\Phi\left(u_{n}\right) \rightarrow \Phi(u)$ in $D^{1,2}\left(\mathbb{R}^{3}\right)$, and

$$
\begin{aligned}
& \int_{\mathbb{R}^{3}} K(x) \phi_{u_{n}} u_{n}^{2} d x \rightarrow \int_{\mathbb{R}^{3}} K(x) \phi_{u} u^{2} d x, \\
& \int_{\mathbb{R}^{3}} K(x) \phi_{u_{n}} u_{n} \psi d x \rightarrow \int_{\mathbb{R}^{3}} K(x) \phi_{u} u \psi d x,
\end{aligned}
$$

for any $\psi \in H^{1}\left(\mathbb{R}^{3}\right)$.

\section{Variational setting}

In this section we describe the variational framework for our problem. Firstly we give the Nehari manifold $M$ corresponding to $I$ :

$$
M=\left\{u \in H^{1}\left(\mathbb{R}^{3}\right) \backslash\{0\}:\left\langle I^{\prime}(u), u\right\rangle=0\right\},
$$

where

$$
\left\langle I^{\prime}(u), u\right\rangle=\|u\|^{2}+\int_{\mathbb{R}^{3}} K(x) \phi_{u} u^{2} d x-\int_{\mathbb{R}^{3}} a(x) u^{4} d x,
$$

and the least energy on $M$ is defined by $c:=\inf _{M} I$.

Lemma 3.1 Let $(\mathrm{K}),\left(\mathrm{a}_{1}\right)$, and either $\left(\mathrm{a}_{2}\right)$ or $\left(\mathrm{a}_{3}\right)$ hold. Then I is coercive on $M$.

Proof For all $u \in M$, we have

$$
I(u)=I(u)-\frac{1}{4}\left\langle I^{\prime}(u), u\right\rangle=\frac{1}{4}\|u\|^{2} .
$$

Hence $\left.I\right|_{M}$ is coercive. 
Next we introduce a set to construct the new method of Nehari manifold as in [21]. Define

$$
\Theta:=\left\{u \in H^{1}\left(\mathbb{R}^{3}\right): \int_{\mathbb{R}^{3}} K(x) \phi_{u} u^{2} d x<\int_{\mathbb{R}^{3}} a(x) u^{4} d x\right\} .
$$

We claim that

$$
\Theta \neq \varnothing .
$$

We shall argue as in [21] to show (3.2), but we need some modifications since $K \in L^{2}\left(\mathbb{R}^{3}\right)$ in this paper, which is different than $L^{\infty}\left(\mathbb{R}^{3}\right)$ considered in [21]. Firstly, we need the following inequality:

Proposition 3.1 (Hardy-Littlewood-Sobolev inequality; see [16]) Let $s, r>1,0<\mu<3$ with $\frac{1}{s}+\frac{\mu}{3}+\frac{1}{r}=2, f \in L^{s}\left(\mathbb{R}^{3}\right)$, and $h \in L^{r}\left(\mathbb{R}^{3}\right)$. There exists a sharp constant $C(s, \mu, r)$, independent off and $h$, such that

$$
\int_{\mathbb{R}^{3}} \int_{\mathbb{R}^{3}} \frac{f(x) h(y)}{|x-y|^{\mu}} d y d x \leq C(s, \mu, r)|f|_{s}|h|_{r} .
$$

Now we prove (3.2). In fact, let $u_{0} \in C_{0}^{\infty}\left(\mathbb{R}^{3},[0,1]\right)$ be such that $u_{0} \equiv 1$ in $B_{r}(0), u_{0} \equiv 0$ in $\mathbb{R}^{3} \backslash B_{2 r}(0)$, where $r$ is to be determined. Then

$$
\int_{\mathbb{R}^{3}} K(x) \phi_{u_{0}} u_{0}^{2} d x=\frac{1}{4 \pi} \int_{\mathbb{R}^{3}} \int_{\mathbb{R}^{3}} \frac{K(x) K(y) u_{0}^{2}(x) u_{0}^{2}(y)}{|x-y|} d y d x \leq C|K|_{2}^{2}\left|u_{0}\right|_{6}^{4} .
$$

Then

$$
\int_{\mathbb{R}^{3}} K(x) \phi_{u_{0}} u_{0}^{2} d x \leq C_{1} r^{2}
$$

Moreover,

$$
\int_{\mathbb{R}^{3}} a(x) u_{0}^{4} d x \geq \inf _{\mathbb{R}^{3}} a \int_{|x| \leq r} d x=C_{2} r^{3} .
$$

Hence we can choose $r$ be so large that $u_{0} \in \Theta$. Then (3.2) follows.

Set

$$
h(t):=I(t u)=\frac{t^{2}}{2}\|u\|^{2}+\frac{t^{4}}{4}\left[\int_{\mathbb{R}^{3}} K(x) \phi_{u} u^{2} d x-\int_{\mathbb{R}^{3}} a(x) u^{4} d x\right] .
$$

Lemma 3.2 Under the assumptions of Lemma 3.1, we have that:

(i) For all $u \in \Theta$, there exists a unique $t_{u}>0$ such that $h^{\prime}(t)>0$ for $0<t<t_{u}$, and $h^{\prime}(t)<0$ for $t>t_{u}$. Moreover, $t_{u} u \in M$ and $I\left(t_{u} u\right)=\max _{t>0} I(t u)$.

(ii) If $u \notin \Theta$, then $t u \notin M$ for any $t>0$.

(iii) For each compact subset $W$ of $\Theta \cap S_{1}$, there exists $C_{W}>0$ such that $t_{w} \leq C_{W}$ for all $w \in W$.

(iv) There exists $\rho>0$ such that $\inf _{S_{\rho}} I>0$ and then $c=\inf _{M} I \geq \inf _{S_{\rho}} I>0$, where $S_{\rho}=\left\{u \in H^{1}\left(\mathbb{R}^{3}\right):\|u\|^{2}=\rho\right\}$.

(v) $\|u\|^{2} \geq 4$ c for all $u \in M$. 
Proof The proof is similar to that given in [21], and we state it for the reader's convenience.

(i) For each $u \in \Theta$, one easily has that $h(t)>0$ when $t$ is sufficiently small, and $h(t)<0$ when $t$ is large enough. Then $h$ has a positive maximum point in $(0, \infty)$. Moreover, the maximum point $t$ satisfies

$$
\|u\|^{2}=t^{2}\left[\int_{\mathbb{R}^{3}} a(x) u^{4} d x-\int_{\mathbb{R}^{3}} K(x) \phi_{u} u^{2} d x\right] .
$$

Then the maximum point is unique, and denoted by $t_{u}$. Therefore, conclusion (i) follows.

(ii) We argue by contradiction. Assume that there exists $t>0$ such that $t u \in M$. Then $\left\langle I^{\prime}(t u), t u\right\rangle=0$. So (3.3) holds. Then $u \in \Theta$. This contradicts the fact that $u \notin \Theta$.

(iii) Suppose that there exist a compact subset $W \subset \Theta \cap S_{1}$ and a sequence $\left\{w_{n}\right\} \subset W$ such that $t_{w_{n}} \rightarrow \infty$. Assume $w \in W$ is such that $w_{n} \rightarrow w$ in $H^{1}\left(\mathbb{R}^{3}\right)$. Then one easily has that

$$
\int_{\mathbb{R}^{3}} K(x) \phi_{w_{n}} w_{n}^{2}-\int_{\mathbb{R}^{3}} a(x) w_{n}^{4} \rightarrow \int_{\mathbb{R}^{3}} K(x) \phi_{w} w^{2}-\int_{\mathbb{R}^{3}} a(x) w^{4}<0 .
$$

So

$$
\frac{I\left(t_{w_{n}} w_{n}\right)}{t_{w_{n}}^{2}}=\frac{1}{2}+\frac{t_{w_{n}}^{2}}{4}\left[\int_{\mathbb{R}^{3}} K(x) \phi_{w_{n}} w_{n}^{2}-\int_{\mathbb{R}^{3}} a(x) w_{n}^{4}\right] \rightarrow-\infty
$$

However, by (3.1), we know that $I\left(t_{w_{n}} w_{n}\right) \geq 0$. This is a contradiction.

(iv) By (2.3), one easily has that there exists $\rho>0$ such that $\inf _{S_{\rho}} I>0$. For any $u \in M$, there is $t>0$ such that $t u \in S_{\rho}$. Note that $I(u) \geq I(t u)$, then $\inf _{S_{\rho}} I \leq \inf _{M} I=c$. Hence $c>0$. In addition, (v) easily follows from (3.1). This ends the proof.

From Lemma 3.2(i), we define the mapping $\hat{m}: \Theta \rightarrow M$ by $\hat{m}(u)=t_{u} u$. In addition, $\forall v \in$ $\mathbb{R}^{+} u$, we have $\hat{m}(v)=\hat{m}(u)$. Let $U:=\Theta \cap S_{1}$. Now we easily infer that $U$ is an open subset of $S_{1}$. Define $m:=\left.\hat{m}\right|_{U}$. Then $m$ is a bijection from $U$ to $M$. Moreover, as in the proof of [20, Proposition 3.1], Lemma 3.2 implies

Lemma 3.3 Under the assumptions of Lemma 3.1, the mapping $m$ is a homeomorphism between $U$ and $M$, and the inverse of $m$ is given by $m^{-1}(u)=\frac{u}{\|u\|}$.

By Lemma 3.3, the least energy $c$ has the following minimax characterization:

$$
c:=\inf _{u \in M} I(u)=\inf _{u \in U} \max _{t \geq 0} I(t u) .
$$

Considering the functional $\Psi: U \rightarrow \mathbb{R}$ given by

$$
\Psi(u):=I(m(u))
$$

and as in [20, Corollary 3.3], we deduce

Lemma 3.4 Under the assumptions of Lemma 3.1, the following results hold:

(1) If $\left\{w_{n}\right\}$ is a PS sequence for $\Psi$, then $\left\{m\left(w_{n}\right)\right\}$ is a PS sequence for I. If $\left\{u_{n}\right\} \subset M$ is a bounded PS sequence for $I$, then $\left\{m^{-1}\left(u_{n}\right)\right\}$ is a PS sequence for $\Psi$. 
(2) $u$ is a critical point of $\Psi$ if and only if $m(u)$ is a nontrivial critical point of I. Moreover, $\inf _{M} I=\inf _{U} \Psi$.

In order to restore the compactness for the PS sequence of $(\mathrm{SP})^{\prime}$, we introduce two equations. If $K(x)=0$, then $(\mathrm{SP})^{\prime}$ turns out to be

$$
-\Delta u+u=a(x) u^{3}
$$

whose functional is

$$
I_{a}(u)=\frac{1}{2}\|u\|^{2}-\frac{1}{4} \int_{\mathbb{R}^{3}} a(x) u^{4} d x,
$$

and the Nehari manifold is

$$
M_{a}=\left\{u \in H^{1}\left(\mathbb{R}^{3}\right) \backslash\{0\}:\left\langle I_{a}^{\prime}(u), u\right\rangle=0\right\} .
$$

When $a(x)=a_{\infty}=1$, (NSE) becomes

$$
-\Delta u+u=u^{3} .
$$

The functional of (NSE) ${ }_{1}$ is

$$
I_{\infty}(u)=I_{1}(u)=\frac{1}{2}\|u\|^{2}-\frac{1}{4} \int_{\mathbb{R}^{3}} u^{4} d x
$$

and the Nehari manifold is

$$
M_{\infty}=M_{1}=\left\{u \in H^{1}\left(\mathbb{R}^{3}\right) \backslash\{0\}:\left\langle I_{1}^{\prime}(u), u\right\rangle=0\right\} .
$$

Moreover, we define the least energy of (NSE) and (NSE) $)_{1}$ by $m_{a}:=\inf _{M_{a}} I_{a}$ and $m_{\infty}=$ $m_{1}:=\inf _{M_{1}} I_{1}$, respectively. We recall some known results about (NSE) and (NSE) ${ }_{1}$; see [8].

Proposition 3.2 Equation (NSE), has a positive ground state $w \in H^{1}\left(\mathbb{R}^{3}\right)$, radially symmetric about the origin, unique up to translations, decaying exponentially, together its derivatives, as $|x| \rightarrow \infty$.

Proposition 3.3 Let $\left(\mathrm{a}_{2}\right)$ hold. Equation (NSE) has a positive ground state $w_{a} \in H^{1}\left(\mathbb{R}^{3}\right)$.

From the above two propositions, we know that

$$
m_{a}=I_{a}\left(w_{a}\right)=\frac{1}{4}\left\|w_{a}\right\|^{2}, \quad m_{1}=I_{1}(w)=\frac{1}{4}\|w\|^{2}=\frac{1}{4}|w|_{4}^{4}
$$

Next we give a compactness lemma, which can be deduced by the argument in $[8$, Lemma 4.1] and Lemma 2.1.

Lemma 3.5 Let $\left\{u_{n}\right\}$ be a bounded (PS) $)_{d}$ sequence for $I$ with $d>0$, then replacing $u_{n}$, if necessary, with a subsequence, there exist $u \in H^{1}\left(\mathbb{R}^{3}\right)$ with $I^{\prime}(u)=0$, a number $k \in \mathbb{N} \cup\{0\}$, functions $w_{1}, w_{2}, \ldots, w_{k}$, and $k$ sequences of points $\left\{y_{n}^{j}\right\} \subset \mathbb{R}^{3}, 1 \leq j \leq k$, such that 
(i) $\left|y_{n}^{j}\right| \rightarrow \infty,\left|y_{n}^{j}-y_{n}^{i}\right| \rightarrow \infty$ as $n \rightarrow \infty$ if $i \neq j$;

(ii) $w_{j} \neq 0$, and $I_{\infty}^{\prime}\left(w_{j}\right)=0$;

(iii) $\left\|u_{n}-u-\sum_{j=1}^{k} w_{j}\left(\cdot-y_{n}^{j}\right)\right\| \rightarrow 0$ as $n \rightarrow \infty$;

(iv) $I\left(u_{n}\right)=I(u)+\sum_{j=1}^{k} I_{\infty}\left(w_{j}\right)+o_{n}(1)$.

Moreover, we agree that in the case $k=0$ the above holds without $w_{j}$.

As in the proof of Lemma 3.1, any (PS) $d$ sequence of $I$ is bounded in $H^{1}\left(\mathbb{R}^{3}\right)$. Then from Lemma 3.5 we easily infer the following two propositions.

Proposition 3.4 Functional I satisfies the PS condition at any level $d<m_{1}$.

Proposition 3.5 Let $\left\{u_{n}\right\}$ be a (PS) $)_{m_{1}}$ sequence. Then either $\left\{u_{n}\right\}$ is relatively compact or the statement of Lemma 3.5 holds with $k=1, u=0$, and $w_{1}=w$, where $w$ is the ground state of $(\mathrm{NSE})_{1}$.

\section{Existence of a ground state}

In this section, we suppose that $\left(\mathrm{a}_{2}\right)$ holds.

Lemma 4.1 Let (1.5) or (1.6) hold. Then $c<m_{\infty}$.

Proof First we assume that (1.5) holds.

Let $w_{a}$ be a ground state of (NSE). Then $w_{a} \in M_{a}$ and $I_{a}\left(w_{a}\right)=m_{a}$. By (1.5) and (2.3), we have that

$$
\begin{aligned}
\int_{\mathbb{R}^{3}} K(x) \phi_{w_{a}} w_{a}^{2} d x & \leq \bar{S}^{-2} S^{-4}|K|_{2}^{2}\left\|w_{a}\right\|^{4} \\
& =4 m_{a} \bar{S}^{-2} S^{-4}|K|_{2}^{2}\left\|w_{a}\right\|^{2}<\left\|w_{a}\right\|^{2}=\int_{\mathbb{R}^{3}} a(x) w_{a}^{4} d x .
\end{aligned}
$$

Then $w_{a} \in \Theta$, and so there exists $t>0$ such that $t w_{a} \in M$. Note that

$$
\begin{aligned}
t^{2} & =\frac{\left\|w_{a}\right\|^{2}}{\int_{\mathbb{R}^{3}} a(x) w_{a}^{4} d x-\int_{\mathbb{R}^{3}} K(x) \phi_{w_{a}} w_{a}^{2} d x} \\
& \leq \frac{\left\|w_{a}\right\|^{2}}{\left\|w_{a}\right\|^{2}-\bar{S}^{-2} S^{-4}|K|_{2}^{2}\left\|w_{a}\right\|^{4}}=\frac{1}{1-\bar{S}^{-2} S^{-4}|K|_{2}^{2} 4 m_{a}}<\frac{m_{1}}{m_{a}} .
\end{aligned}
$$

Hence,

$$
c \leq I\left(t w_{a}\right)=\frac{1}{4} t^{2}\left\|w_{a}\right\|^{2}=t^{2} m_{a}<m_{1} .
$$

Next we assume (1.6) holds.

Let $w$ be the ground state of (NSE) ${ }_{1}$. By (1.6), we get $w \in \Theta$. Then there exists $t>0$ such that $t w \in M$. First we show that $t<1$. Indeed, if $t \geq 1$, using $\|w\|^{2}=|w|_{4}^{4}$ we infer that

$$
\begin{aligned}
0 & =t^{2}\|w\|^{2}+t^{4} \int_{\mathbb{R}^{3}} K(x) \phi_{w} w^{2} d x-t^{4} \int_{\mathbb{R}^{3}} a(x)|w|^{4} d x \\
& \leq t^{4} \int_{\mathbb{R}^{3}} K(x) \phi_{w} w^{2} d x-t^{4} \int_{\mathbb{R}^{3}}(a(x)-1)|w|^{4} d x .
\end{aligned}
$$


This contradicts (1.6). So $t<1$. Then

$$
c \leq I(t w)=I(t w)-\frac{1}{4}\left\langle I^{\prime}(t w), t w\right\rangle=\frac{1}{4} t^{2}\|w\|^{2}=t^{2} m_{1}<m_{1},
$$

completing the proof.

Proof of Theorem 1.1 Assume that $w_{n} \in U$ satisfies that $\Psi\left(w_{n}\right) \rightarrow \inf _{U} \Psi$. By the Ekeland variational principle, we may suppose that $\Psi^{\prime}\left(w_{n}\right) \rightarrow 0$. From Lemma 3.4 it follows that $I^{\prime}\left(u_{n}\right) \rightarrow 0$ and $I\left(u_{n}\right) \rightarrow c$, where $u_{n}=m\left(w_{n}\right) \in M$. Then by Lemma 4.1 and Proposition 3.4, there exists $u \in H^{1}\left(\mathbb{R}^{3}\right)$ such that $u_{n} \rightarrow u$ in $H^{1}\left(\mathbb{R}^{3}\right)$. Then $I(u)=c$ and $I^{\prime}(u)=0$. This ends the proof.

\section{Existence of a bound state}

Throughout this section we suppose that $\left(\mathrm{a}_{3}\right)$ holds. First we show that, under $\left(\mathrm{a}_{3}\right)$, problem $(\mathrm{SP})^{\prime}$ cannot be solved by minimization.

Lemma 5.1 $c=m_{1}$ and $c$ is not attained.

Proof First we show that $c \geq m_{1}$. For all $u \in U$, by the standard argument, there exists $t_{0}>0$ such that $t_{0} u \in M_{1}$. By $K \geq 0$ and $a(x) \leq 1$, we have

$$
m_{1} \leq I_{1}\left(t_{0} u\right) \leq I\left(t_{0} u\right) \leq \max _{t>0} I(t u)
$$

Using (3.4), we get $m_{1} \leq \inf _{u \in U} \max _{t>0} I(t u)=c$.

Below we shall show that $c \leq m_{\infty}$. Indeed, set $w_{n}(x)=w\left(x-z_{n}\right)$, where $w$ the positive solution centered at zero of (NSE) $)_{1}$, and $\left|z_{n}\right| \rightarrow \infty$ as $n \rightarrow \infty$.

In fact, since $w_{n} \rightarrow 0$ in $H^{1}\left(\mathbb{R}^{3}\right)$, by Lemma 2.1(4) we have that

$$
\int_{\mathbb{R}^{3}} K(x) \phi_{w_{n}} w_{n}^{2} d x \rightarrow 0, \quad \int_{\mathbb{R}^{3}} a(x) w_{n}^{4} d x \rightarrow \int_{\mathbb{R}^{3}} w^{4} d x,
$$

so for $n$ large enough, we have

$$
\int_{\mathbb{R}^{3}} K(x) \phi_{w_{n}} w_{n}^{2} d x<\int_{\mathbb{R}^{3}} a(x) w_{n}^{4} d x
$$

and then

$$
w_{n} \in \Theta \text {. }
$$

Considering $u_{n}=t_{n} w_{n} \in M$, we claim that

$$
I\left(u_{n}\right) \rightarrow m_{1} \quad \text { as } n \rightarrow \infty .
$$

Since $t_{n} w_{n} \in M$, we get

$$
\frac{1}{t_{n}^{2}}\left\|w_{n}\right\|^{2}+\int_{\mathbb{R}^{3}} K(x) \phi_{w_{n}} w_{n}^{2} d x=\int_{\mathbb{R}^{3}} a(x) w_{n}^{4} d x
$$


By (5.1), we have

$$
\frac{1}{t_{n}^{2}}\|w\|^{2}=\int_{\mathbb{R}^{3}} w^{4} d x+o_{n}(1)
$$

Observe that

$$
\|w\|^{2}=\int_{\mathbb{R}^{3}} w^{4} d x
$$

then we have $t_{n}=1+o_{n}(1)$, and, using (5.1) again, infer

$$
I\left(u_{n}\right)=I\left(t_{n} w_{n}\right) \rightarrow \frac{1}{4}\|w\|^{2}=m_{1} .
$$

Then (5.3) follows and so $c \leq m_{1}$. Hence, $c=m_{1}$.

Finally, arguing by contradiction, we assume that there exists $\bar{u} \in M$ such that $I(\bar{u})=c=$ $m_{1}$. By a standard argument, there exists $\xi>0$ such that $\xi \bar{u} \in M_{1}$, and it is easy to see that $\xi \leq 1$. Then

$$
m_{1} \leq I_{1}(\xi \bar{u})=\frac{1}{4}\|\xi \bar{u}\|^{2} \leq \frac{1}{4}\|\bar{u}\|^{2}=I(\bar{u})-\frac{1}{4}\left\langle I^{\prime}(\bar{u}), \bar{u}\right\rangle=c=m_{1} .
$$

Therefore, $\xi=1, \bar{u} \in M_{1}$ and $I_{1}(\bar{u})=I(\bar{u})=m_{1}$. Then

$$
\frac{1}{2}\|\bar{u}\|^{2}-\frac{1}{4} \int_{\mathbb{R}^{3}}|\bar{u}|^{4} d x=\frac{1}{2}\|\bar{u}\|^{2}+\frac{1}{4} \int_{\mathbb{R}^{3}} K(x) \phi_{\bar{u}} \bar{u}^{2} d x-\frac{1}{4} \int_{\mathbb{R}^{3}} a(x)|\bar{u}|^{4} d x .
$$

Since $K \geq 0$ and $a(x) \leq 1$, we get

$$
\int_{\mathbb{R}^{3}} K(x) \phi_{\bar{u}} \bar{u}^{2} d x=0
$$

Hence, by the uniqueness of the family realizing $m_{1}$, we have

$$
\bar{u}(\cdot)=w_{z}:=w(\cdot-z)>0,
$$

for some $z \in \mathbb{R}^{3}$. Therefore,

$$
\int_{\mathbb{R}^{3}} K(x) \phi_{\bar{u}} \bar{u}^{2} d x=\int_{\mathbb{R}^{3}} K(x) \phi_{w_{z}} w_{z}^{2} d x>0,
$$

which contradicts (5.4).

By the previous lemma, we can only hope to find critical points of $I$ at a level higher than $m_{1}$. Similar to [8, Lemma 6.2], we have

Lemma 5.2 Functional I satisfies the $(\mathrm{PS})_{d}$ condition for all $d \in\left(m_{1}, 2 m_{1}\right)$.

Below we shall use the notion of a barycenter to build a suitable min-max level which belongs to $\left(m_{1}, 2 m_{1}\right)$. Firstly we recall the definition of the barycenter of a function $u \in$ 
$H^{1}\left(\mathbb{R}^{3}\right) \backslash\{0\}$ given in [7]. Setting

$$
\begin{aligned}
& \mu(u)(x)=\frac{1}{\left|B_{1}(0)\right|} \int_{B_{1}(x)}|u(y)| d y, \quad \mu(u) \in L^{\infty}\left(\mathbb{R}^{3}\right) \text { and is continuous, } \\
& \hat{u}(x)=\left[\mu(u)(x)-\frac{1}{2} \max \mu(u)(x)\right]^{+}, \quad \hat{u} \in C_{0}\left(\mathbb{R}^{3}\right),
\end{aligned}
$$

we define the barycenter $\beta: H^{1}\left(\mathbb{R}^{3}\right) \backslash\{0\} \rightarrow \mathbb{R}^{3}$ by

$$
\beta(u)=\frac{1}{|\hat{u}|_{1}} \int_{\mathbb{R}^{3}} x \hat{u}(x) d x \in \mathbb{R}^{3} .
$$

Since $\hat{u}$ has a compact support, $\beta$ is well defined. Moreover, the following properties hold:

\section{Lemma 5.3}

(1) $\beta$ is continuous in $H^{1}\left(\mathbb{R}^{3}\right) \backslash\{0\}$.

(2) If $u$ is a radial function, $\beta(u)=0$.

(3) For all $t \neq 0$ and for all $u \in H^{1}\left(\mathbb{R}^{3}\right) \backslash\{0\}, \beta(t u)=\beta(u)$.

(4) Given $z \in \mathbb{R}^{3}$ and setting $u_{z}(x)=u(x-z), \beta\left(u_{z}\right)=\beta(u)+z$.

Let us define

$$
b_{0}:=\inf \{I(u): u \in M, \beta(u)=0\}
$$

By Lemmas 3.3 and 5.3(3), it is easy to see that

$$
b_{0}:=\inf \{\Psi(v): v \in U, \beta(v)=0\}
$$

Lemma $5.4 b_{0}>c$.

Proof Clearly, $b_{0} \geq c$. Assume that $b_{0}=c$. By (5.5), there exists $v_{n} \in U$ such that $\beta\left(v_{n}\right)=0$, and $\Psi\left(v_{n}\right) \rightarrow b_{0}=c=m_{\infty}$. By Lemma 3.4(2), $\Psi\left(v_{n}\right) \rightarrow \inf _{U} \Psi$. By the Ekeland variational principle, we may assume that $\Psi^{\prime}\left(v_{n}\right) \rightarrow 0$. Set $u_{n}=m\left(v_{n}\right)=t_{v_{n}} v_{n}$. Then $I\left(u_{n}\right) \rightarrow c=m_{\infty}$, $I^{\prime}\left(u_{n}\right) \rightarrow 0$ and $\beta\left(u_{n}\right)=0$ by Lemma 5.3(3). Then Proposition 3.5 and Lemma 5.1 yield

$$
u_{n}(x)=w\left(x-z_{n}\right)+o_{n}(1)
$$

where $\left|z_{n}\right| \rightarrow+\infty$ and $w$ is the positive solution of (NSE) $)_{1}$. Then

$$
\beta\left(u_{n}\left(x+z_{n}\right)\right)=\beta\left(u_{n}\right)-z_{n}=-z_{n} .
$$

However,

$$
\beta\left(u_{n}\left(x+z_{n}\right)\right)=\beta\left(w(x)+o_{n}(1)\right)=\beta(w(x))=0 .
$$

This is a contradiction since $\left|z_{n}\right| \rightarrow+\infty$. 
Now let us define the operator $\Gamma: \mathbb{R}^{3} \rightarrow M$ as $\Gamma[z](x)=t_{z} w(x-z)$, where $w$ is the positive solution of $(\mathrm{NSE})_{\infty}$ and $t_{z}$ is chosen such that $\Gamma[z] \in M$. As (5.2), $w(x-z) \in \Theta$ for any $z \in \mathbb{R}^{3}$. Then $\Gamma$ is well defined. From Lemma 5.3 it follows that

$$
\beta(\Gamma[z])=\beta\left(t_{z} w(x-z)\right)=\beta(w(x-z))=z .
$$

Lemma 5.5 Assume that (1.7) holds. Then $I(\Gamma[z])<2 m_{1}$.

Proof Since $\Gamma[z] \in M$, we have

$$
I(\Gamma[z])=\frac{1}{4}\|\Gamma[z]\|^{2}=\frac{1}{4} t_{z}^{2}\left\|w_{z}\right\|^{2}=\frac{1}{4} t_{z}^{2}\|w\|^{2}=t_{z}^{2} m_{1} .
$$

It suffices to show $t_{z}^{2}<2$. Note that

$$
t_{z}^{2}\left\|w_{z}\right\|^{2}+t_{z}^{4} \int_{\mathbb{R}^{3}} K(x) \phi_{w_{z}} w_{z}^{2} d x=t_{z}^{4} \int_{\mathbb{R}^{3}} a(x) w_{z}^{4} d x
$$

Then

$$
\begin{aligned}
t_{z}^{2} & =\frac{\left\|w_{z}\right\|^{2}}{\int_{\mathbb{R}^{3}} a(x) w_{z}^{4} d x-\int_{\mathbb{R}^{3}} K(x) \phi_{w_{z}} w_{z}^{2} d x} \\
& <\frac{\left\|w_{z}\right\|^{2}}{\inf _{x \in \mathbb{R}^{3}} a(x)\left\|w_{z}\right\|^{2}-\bar{S}^{-2} S^{-4}|K|_{2}^{2}\left\|w_{z}\right\|^{4}} \\
& =\frac{1}{\inf _{x \in \mathbb{R}^{3}} a(x)-\bar{S}^{-2} S^{-4}|K|_{2}^{2} 4 m_{1}}<2 .
\end{aligned}
$$

This completes the proof.

Now we are ready to prove Theorem 1.2.

Proof of Theorem 1.2 Arguing as when deriving (5.3), we obtain $\lim _{|z| \rightarrow+\infty} I(\Gamma[z])=m_{1}$. Combining with Lemmas 5.4 and 5.1, there exists $\rho>0$ such that

$$
\max _{|z|=\rho} I(\Gamma[z])<b_{0}
$$

Set

$$
Q=\Gamma\left(\bar{B}_{\rho}(0)\right), \quad S=\{u \in M: \beta(u)=0\} .
$$

We claim that $S$ and $\partial Q$ link, that is,

(i) $\partial Q \cap S=\varnothing$;

(ii) $h(Q) \cap S \neq \varnothing, \quad \forall h \in \mathcal{H}=\left\{h \in C(Q, M):\left.h\right|_{\partial Q}=i d\right\}$.

Firstly, we show (5.8)(i). Indeed, if $u \in \partial Q$, then $u=\Gamma[z],|z|=\rho$. By (5.6), we get $\beta(u)=$ $\beta(\Gamma[z])=z$, and so $u \notin S$. Next we show (5.8)(ii). Consider $h \in \mathcal{H}$ and define

$$
T: \bar{B}_{\rho}(0) \rightarrow \mathbb{R}^{3}, \quad T(z)=\beta \circ h \circ \Gamma[z] .
$$


This $T$ is a continuous function. Moreover, for all $|z|=\rho, \Gamma[z] \in \partial Q$, we have $h \circ \Gamma[z]=$ $\Gamma[z]$. Then $T(z)=z$. Brouwer fixed point theorem implies that there exists $z \in B_{\rho}(0)$ such that $T(z)=0$, and then $h(\Gamma[z]) \in S$. Thus $h(Q) \cap S \neq \varnothing$.

By (5.7), we get $b_{0}=\inf _{S} I>\max _{\partial Q} I$. Define

$$
d:=\inf _{h \in \mathcal{H}} \max _{u \in Q} I(h(u)) .
$$

By (5.8)(ii), $d \geq b_{0}>c=m_{1}$. Moreover, taking $h=i d$ and using Lemma 5.5, we have that $d<2 m_{1}$. By Lemma 5.2, the PS condition holds in $\left(m_{1}, 2 m_{1}\right)$. Then the linking theorem [18, Theorem 8.4] implies that $d$ is a critical value of $I$. This ends the proof.

\section{Acknowledgements}

The authors would like to express sincere thanks to the anonymous referees for their carefully reading of the manuscript and valuable comments and suggestions. The work is supported by the National Natural Science Foundation of China (No. 11671077).

\section{Funding}

The paper is supported by the National Natural Science Foundation of China (No. 11671077).

\section{Availability of data and materials}

Not applicable.

\section{Competing interests}

The authors declare that they have no competing interests.

\section{Authors' contributions}

The authors declare that this study was independently finished. All authors read and approved the final manuscript.

\section{Authors' information}

Not applicable.

\section{Publisher's Note}

Springer Nature remains neutral with regard to jurisdictional claims in published maps and institutional affiliations.

Received: 27 March 2019 Accepted: 30 June 2019 Published online: 12 July 2019

\section{References}

1. Alves, C.O., Souto, M.A.S., Soares, S.H.M.: Schrödinger-Poisson equations without Ambrosetti-Rabinowitz condition. J. Math. Anal. Appl. 377, 584-592 (2011)

2. Ambrosetti, A.: On Schrödinger-Poisson systems. Milan J. Math. 76, 257-274 (2008)

3. Ambrosetti, A., Ruiz, D.: Multiple bound states for the Schrödinger-Poisson problem. Commun. Contemp. Math. 10 391-404 (2008)

4. Azzollini, A., Pomponio, A.: Ground state solutions for the nonlinear Schrödinger-Maxwell equations. J. Math. Anal. Appl. 345, 90-108 (2008)

5. Benci, V., Fortunato, D.: An eigenvalue problem for the Schrödinger-Maxwell equations. Topol. Methods Nonlinear Anal. 11, 283-293 (1998)

6. Cerami, G., Molle, R.: Positive bound state solutions for some Schrödinger-Poisson systems. Nonlinearity 29 3103-3119 (2016)

7. Cerami, G., Passaseo, D.: The effect of concentrating potentials in some singularly perturbed problems. Calc. Var. Partial Differ. Equ. 17, 257-281 (2003)

8. Cerami, G., Vaira, G.: Positive solutions for some nonautonomous Schrödinger-Poisson systems. J. Differ. Equ. 248, $521-543(2010)$

9. D’Aprile, T., Mugnai, D.: Solitary waves for nonlinear Klein-Gordon-Maxwell and Schrödinger-Maxwell equations. Proc. R. Soc. Edinb. A 134, 893-906 (2004)

10. D'Aprile, T., Wei, J.: On bound states concentrating on spheres for the Maxwell-Schrödinger equation. SIAM J. Math. Anal. 37, 321-342 (2005)

11. D'Avenia, P.: Non-radially symmetric solutions of nonlinear Schrödinger equation coupled with Maxwell equations. Adv. Nonlinear Stud. 2, 177-192 (2002)

12. Fang, X.D., Szulkin, A.: Multiple solutions for a quasilinear Schrödinger equation. J. Differ. Equ. 254, 2015-2032 (2013)

13. Furtado, M.F., Maia, L.A., Medeiros, E.S.: A note on the existence of a positive solution for a non-autonomous Schrödinger-Poisson system. In: Analysis and Topology in Nonlinear Differential Equations. Progr. Nonlinear Differential Equations Appl., vol. 85, pp. 277-286. Springer, Cham (2014)

14. Li, F.Y., Li, Y.H., Shi, J.P.: Existence of positive solutions to Schrödinger-Poisson type systems with critical exponent. Commun. Contemp. Math. 16, 1450036 (2014) 28 pp. 
15. Li, G.B., Peng, S.J., Yan, S.: Infinitely many positive solutions for the nonlinear Schrödinger-Poisson system. Commun. Contemp. Math. 12, 1069-1092 (2010)

16. Lieb, E.H., Loss, M.: Analysis, Graduate Studies in Mathematics, Am. Math. Soc., Providence (2001)

17. Ruiz, D.: The Schrödinger-Poisson equation under the effect of a nonlinear local term. J. Funct. Anal. 237, 655-674 (2006)

18. Struwe, M.: Variational Methods. Springer, Berlin (1996)

19. Sun, J.T., Chen, H.B., Nieto, J.: On ground state solutions for some non-autonomous Schrödinger-Poisson systems. J. Differ. Equ. 252, 3365-3380 (2012)

20. Szulkin, A., Weth, T.: The method of Nehari manifold. In: Gao, D.Y., Motreanu, D. (eds.) Handbook of Nonconvex Analysis and Applications, pp. 597-632. International Press, Boston (2010)

21. Zhang, H., Zhang, F.B., Xu, J.X.: Ground states for Schrödinger-Poisson systems with three growth terms. Electron. J. Differ. Equ. 2014, No. 253, 1-13 (2014)

22. Zhang, X., Ma, S.W., Xie, Q.L.: Bound state solutions of Schrödinger-Poisson system with critical exponent. Discrete Contin. Dyn. Syst. 37, 605-625 (2017)

23. Zhao, L.G., Zhao, F.K.: On the existence of solutions for the Schrödinger-Poisson equations. J. Math. Anal. Appl. 346 155-169 (2008)

Submit your manuscript to a SpringerOpen ${ }^{\odot}$ journal and benefit from:

- Convenient online submission

- Rigorous peer review

- Open access: articles freely available online

- High visibility within the field

- Retaining the copyright to your article

Submit your next manuscript at $\boldsymbol{\nabla}$ springeropen.com 